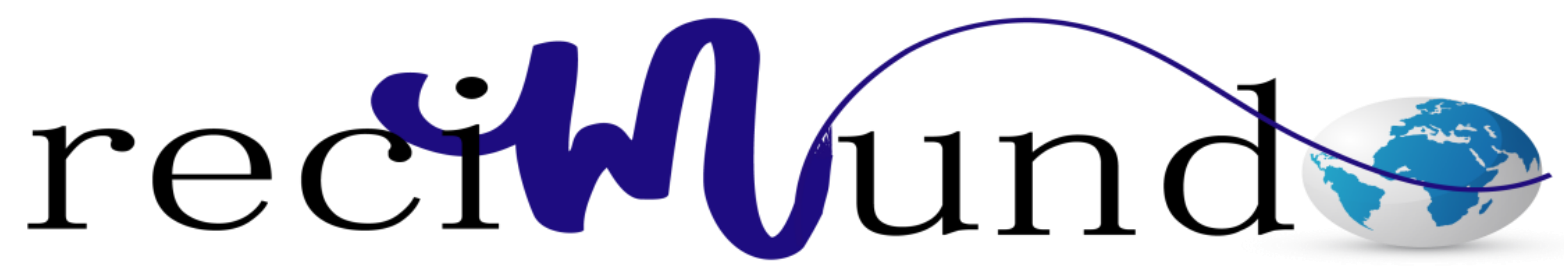

Revista Cientifica Mundo de la Investígación y el Conocimiento

Karla Lissette Flores Flores ${ }^{a}$; Luis Eduardo López Ávila ${ }^{\text {b; }}$ Linda Beatriz Dávila Solorzano ${ }^{c}$; Stalin Josue Alvarado Fiallo ${ }^{d}$

Hemorragia digestiva alta asociada a AINES

Upper digestive hemorrhage associated with NSAIDs

Revista Científica Mundo de la Investigación y el Conocimiento. Vol. 3 núm.3. Esp., noviembre, ISSN: 2588-073X, 2019, pp. 128-145

DOI: $10.26820 /$ recimundo/3.(3.Esp).noviembre.2019.128-145

URL: http://recimundo.com/index.php/es/article/view/641

Código UNESCO: 3205 Medicina Interna

Tipo de Investigación: Artículo de Revisión

(c) RECIMUNDO; Editorial Saberes del Conocimiento, 2019

Recibido: 15/09/2019

Aceptado: 23/10/2019

Publicado: 30/11/2019

Correspondencia: floresfkl@gmail.com

\footnotetext{
a. Médico; Investigadora Independiente; Guayaquil, Ecuador; floresfkl@ gmail.com

b. Médico; Investigador Independiente; Guayaquil, Ecuador; edu_31189@ hotmail.com

c. Médico; Investigadora Independiente; Guayaquil, Ecuador; lindbed_2605@ @otmail.es

d. Médico; Investigador Independiente; Guayaquil, Ecuador; stalin.af@hotmail.com
} 


\section{Hemorragia digestiva alta asociada a AINES}

Vol. 3, núm. 3 Esp., (2019)

Karla Lissette Flores Flores; Luis Eduardo López Ávila; Linda Beatriz Dávila Solorzan; Stalin

Josue Alvarado Fiallo

\section{RESUMEN}

Los antinflamatorios no esteroideos (AINES) son fármacos de uso frecuente en la práctica clínica y aunque en forma general son bien tolerados y tienen una gran variedad de posibilidades terapéuticas, cabe mencionar que pueden ocasionar serios efectos adversos gastrointestinales que implican morbilidad seria e incluso mortalidad tales como "la gastropatía por AINES". La presente investigación se realizó en el marco de una investigación documental con una metodología de revisión con el fin de exponer los efectos secundarios a nivel gastrointestinal ocasionados por la administración excesiva de los fármacos denominados AINES, así como también los factores de riesgo y la posible predisposición a padecer de este tipo de afecciones. En general, la literatura indica que al menos del 10 al 20\% de los pacientes usuarios de AINES presentan dispepsia, aunque la prevalencia puede variar entre el 5 y el 50\%. En conclusión, se consiente que la aplicación de métodos de vigilancia intensiva por personal capacitado resulta fundamental para el desarrollo y fortalecimiento de la fármaco-vigilancia (FV) y la sensibilización y educación de los profesionales de la salud y los pacientes. La hemorragia digestiva alta (HDA) es, con una gran diferencia, el efecto indeseado más grave y frecuente de los AINES. Como consecuencia, dada la alta frecuencia de la asociación de ingesta de AINES y HDA aguda, parece prudente que los especialistas sean más cautos en la indicación de AINES, descartar el alcoholismo y la hipertensión arterial y confirmar que no existe antecedente ulceroso. Como alternativa a estos efectos indeseados usar nuevas drogas anti-inflamatorias que tienen menor efecto dañino sobre la mucosa gastrointestinal.

Palabras Claves: Antinflamatorio; Esteroideos; Hemorragia; Digestiva; Fármacos. 


\section{Hemorragia digestiva alta asociada a AINES}

Vol. 3, núm. 3 Esp., (2019)

Karla Lissette Flores Flores; Luis Eduardo López Ávila; Linda Beatriz Dávila Solorzan; Stalin Josue Alvarado Fiallo

\section{ABSTRACT}

Nonsteroidal anti-inflammatory drugs (NSAIDs) are drugs often used in clinical practice and although in general they are well tolerated and have a wide variety of therapeutic possibilities, it is worth mentioning that they can cause serious gastrointestinal adverse effects that involve serious morbidity and even mortality such as "NSAID gastropathy." The present investigation was carried out within the framework of a documentary investigation with a review methodology in order to expose the side effects at the gastrointestinal level caused by the excessive administration of the drugs called NSAIDs, as well as the risk factors and the possible predisposition to suffer from these types of conditions. In general, the literature indicates that at least 10 to $20 \%$ of patients using NSAIDs have dyspepsia, although the prevalence can vary between 5 and 50\%. In conclusion, it is agreed that the application of intensive surveillance methods by trained personnel is essential for the development and strengthening of drug surveillance (VF) and the awareness and education of health professionals and patients. High digestive hemorrhage (HDA) is, by far, the most serious and frequent unwanted effect of NSAIDs. As a consequence, given the high frequency of the intake association of NSAIDs and acute HDA, it seems prudent for specialists to be more cautious in the indication of NSAIDs, rule out alcoholism and arterial hypertension and confirm that there is no ulcerative history. As an alternative to these unwanted effects, use new anti-inflammatory drugs that have less harmful effect on the gastrointestinal mucosa.

Keywords: Anti-inflammatory; Steroidal; Hemorrhage; Digestive; Drugs. 


\section{Hemorragia digestiva alta asociada a AINES}

Vol. 3, núm. 3 Esp., (2019)

Karla Lissette Flores Flores; Luis Eduardo López Ávila; Linda Beatriz Dávila Solorzan; Stalin

Josue Alvarado Fiallo

\section{Introducción.}

El término gastropatía por AINES fue introducido en 1987 con la finalidad de diferenciar a la úlcera producida por estos agentes de la úlcera péptica clásica, así como para distinguirla de otras gastropatías como la hipertensiva que se presenta en el cirrótico y de las alteraciones de las gastritis comunes en donde predomina el componente inflamatorio y no el que caracteriza al producido por los AINE. (Castellanos, Solis, Faure, \& Villaurrutia, 2014)

Los antinflamatorios no esteroideos (AINES), son los medicamentos más utilizados mundialmente para el alivio de dolor e inflamación asociados con artritis reumatoide (AR) y otros procesos osteomusculares. Sin embargo, este beneficio está estrechamente asociado con efectos adversos, principalmente con el riesgo de desarrollar úlcera péptica y complicaciones graves, como hemorragia y perforación1. Hay una gran diversidad en las alteraciones gastrointestinales causadas por el uso de AINES, algunas muy inespecíficas y con grandes diferencias en las poblaciones estudiadas, diferentes medicamentos, distintas dosis y períodos también muy variables, por lo que la prevalencia reportada de efectos adversos varía en forma importante. En general, al menos del 10 al 20\% de los pacientes usuarios de AINES presentan dispepsia, aunque la prevalencia puede variar entre el 5 y el 50\%. Sin embargo, dichos síntomas no correlacionan con la apariencia endoscópica y la severidad del daño a la mucosa, ya que más del $40 \%$ de las personas con evidencia endoscópica de gastritis erosiva cursa asintomático y, al contrario, cerca del $50 \%$ de los pacientes con dispepsia tiene una mucosa gástrica de apariencia normal. (Galvan \& Neri, 2006)

Los AINES constituyen un grupo fármacos que tiene una gran variedad de posibilidades 


\section{Hemorragia digestiva alta asociada a AINES}

Vol. 3, núm. 3 Esp., (2019)

Karla Lissette Flores Flores; Luis Eduardo López Ávila; Linda Beatriz Dávila Solorzan; Stalin Josue Alvarado Fiallo

terapéuticas, pero pueden presentar numerosos efectos adversos, entre los que destaca como un limitante de su uso "la gastropatía por AINES". Esta se define como el conjunto de síntomas sin una lesión aparente y de lesiones con o sin síntomas inducidos por los AINES en la mucosa digestiva. Las manifestaciones digestivas son amplias, y abarcan desde síntomas leves como petequias, equimosis y erosiones, que normalmente son asintomáticos, hasta situaciones más graves de hemorragia digestiva alta (HDA) y perforación. (Marco, et al., 2004)

Los antinflamatorios no esteroideos (AINES) son fármacos de uso frecuente en la práctica clínica y aunque en forma general son bien tolerados, pueden ocasionar serios efectos adversos gastrointestinales que implican morbilidad seria e incluso mortalidad. Este aspecto a menudo no se toma en cuenta al indicarlos. (Roblero \& Valenzuela, 2002)

Los efectos adversos pueden ser prevenidos si se consideran las características del paciente, especialmente los que presentan factores de riesgo tales como, la edad (mayor de 65 años), antecedentes de úlcera o hemorragia digestiva, en tratamiento con anticoagulantes, dosis altas o varios AINES, uso de corticoides, patología asociada grave). (Franco)

La presente investigación se realizó con el fin de exponer los efectos secundarios a nivel gastrointestinal ocasionados por la administración excesiva de los fármacos denominados AINES, así como también los factores de riesgo y la posible predisposición a padecer de este tipo de afecciones. 


\section{Hemorragia digestiva alta asociada a AINES}

Vol. 3, núm. 3 Esp., (2019)

Karla Lissette Flores Flores; Luis Eduardo López Ávila; Linda Beatriz Dávila Solorzan; Stalin

Josue Alvarado Fiallo

\section{Materiales y Métodos.}

Las herramientas y materiales a los que se recurrieron para el desarrollo de este trabajo investigativo son los siguientes: computadores personales con conexión a internet y un cúmulo de documentos científico-académicos diversos, con los que se espera aportar información de calidad y lo más actualizada respecto a la Hemorragia digestiva causada por AINES. Se escoge un diseño documental, de tipo no experimental, a nivel descriptivo y bajo una metodología de revisión.

Esta investigación se enfoca en la búsqueda de literatura científico-académica que, por una parte, está disponible en determinadas bases de datos, entre las que figuran: MedlinePlus, PubMed, Biblioteca Virtual de la Salud (BVS), SciELO, Dialnet y ELSEVIER, Cochrane, entre otras; y por la otra parte, mediante el uso del material que físicamente disponible tanto en la biblioteca de la Universidad como en las particulares, representando todo ello parte esencial del proceso investigativo y de comprensión que facilita la síntesis de la mejor evidencia disponible, y a su vez representando con todo esto los resultados esperados.

A fines de identificar y referir cuáles serían las directrices que actualmente están disponibles en el basto mundo de las fuentes bibliográficas en cuanto al tratamiento de estos efectos secundarios asociados a fármacos AINES, principalmente se han considerado rescatar: artículos científicos, guías clínicas, e-books, ensayos clínicos, consensos, revisiones sistemáticas con o sin meta-análisis, boletines y/o folletos de instituciones privadas, formales u oficiales, tesis de posgrado y doctorado, noticias científicas y demás documentos e informaciones asumidos bajo propio criterio del equipo investigador como de interés y considerable valor de la evidencia 


\section{Hemorragia digestiva alta asociada a AINES}

Vol. 3, núm. 3 Esp., (2019)

Karla Lissette Flores Flores; Luis Eduardo López Ávila; Linda Beatriz Dávila Solorzan; Stalin Josue Alvarado Fiallo

científica, publicados preferiblemente en los últimos 10 años (incluyendo el presente), es decir, desde 2009 hasta el 2019, salvo algunas excepciones.

Se desestimaron estudios de cohorte, casos y controles, series y reportes de casos, editoriales, cartas al editor y otros tipos de materiales bibliográficos que, a juicio de este equipo, se consideraron de escaso valor científico-académico o de bajo nivel de evidencia percibido en sus contenidos.

El resultado obtenido tras la búsqueda en dichas bases de datos, en conjunto ascendió a más 1.260 referencias bibliográficas, lo que evidenció la existencia de suficiente contenido bibliográfico desarrollado en base a esta temática, sin embargo, fue necesaria la depuración de los mismos en función de los objetivos previamente planteados y a razones tales como: idioma español, relevancia, correlación temática y fecha de publicación en el lapso antes mencionado, fundamentalmente.

Paralelamente, se realizó una búsqueda sin limitación cronológica ni de nivel de evidencia para identificar información igualmente relevante, necesaria para el desarrollo de la presente temática y revisión, siendo a partir de allí que el equipo investigador le da la correspondiente lectura crítica y análisis interpretativo a toda la evidencia en definitiva seleccionada, resultando dicho proceso en el fundamento de las ideas aquí expresadas de manera consensuada.

La inclusión o exclusión de cada una de las referencias en la presente revisión se definió igualmente por el consenso todos los participantes en este proceso investigativo, y de la misma manera fueron resueltas las decisiones desiguales. 


\section{Hemorragia digestiva alta asociada a AINES}

Vol. 3, núm. 3 Esp., (2019)

Karla Lissette Flores Flores; Luis Eduardo López Ávila; Linda Beatriz Dávila Solorzan; Stalin

Josue Alvarado Fiallo

\section{Resultados.}

Los efectos adversos más importantes y conocidos de los AINES son los que conllevan riesgos gastrointestinales, cardiovasculares y renales, aunque también pueden ocasionar otros problemas como hepatotoxicidad o reacciones de hipersensibilidad graves. Numerosos estudios han evaluado la toxicidad gastrointestinal de los AINES, siendo la HDA el efecto indeseable grave más frecuente descrito, su riesgo aumenta cuanto mayor es la dosis empleada. En un estudio realizado en 2004 en España e Italia, la incidencia de HDA fue de 4 casos por 10.000 individuos-año. Los resultados indicaban que el 38\% de los casos, eran atribuibles a los AINES. En dicho estudio se confirmaron los riesgos elevados de HDA asociados a ketorolaco, piroxicam, indometacina, ketoprofeno, naproxeno y ácido acetilsalicílico (AAS), incluso utilizados a dosis bajas. Otros AINES como el dexketoprofeno, meloxicam y rofecoxib (fármaco retirado) presentaban un riesgo moderado, mientras que el uso de aceclofenaco, ibuprofeno $y$ diclofenaco, a dosis bajas, se asoció a un riesgo bajo de HDA. El empleo de celecoxib se relacionó con un bajo riesgo, pero esta información hay que tomarla con cautela pues en el estudio había muy pocos pacientes en tratamiento con este fármaco. (Aranguren, Elizondo, \& Azparren, 2016)

Se ha evidenciado endoscópicamente que cerca de la mitad de los pacientes que reciben crónicamente AINES presentan erosiones gástricas, y entre un $15 \%$ y un $30 \%$ de los mismos, presentan úlceras gastroduodenales. Sin embargo, la incidencia de eventos clínicos gastrointestinales (GI) causados por la administración de estas drogas es mucho menor. Así se ha evidenciado la ocurrencia de eventos clínicos GI "altos" en aproximadamente 5\% de los pacientes que reciben AINES, en tanto se han observado complicaciones severas (perforación, 


\section{Hemorragia digestiva alta asociada a AINES}

Vol. 3, núm. 3 Esp., (2019)

Karla Lissette Flores Flores; Luis Eduardo López Ávila; Linda Beatriz Dávila Solorzan; Stalin Josue Alvarado Fiallo

obstrucción, sangrado significativo) en cerca del 1,5\% de estos pacientes. Se ha demostrado que existen algunos factores que incrementan el riesgo de presentar complicaciones GI asociadas con la administración de AINES. (Bai \& Smecuol, 2004)

\section{Alteraciones digestivas por AINES}

Estos medicamentos, por su mecanismo de acción, pueden producir efectos no deseables en el organismo y, muy especialmente, en el aparato digestivo. Entre ellos cabe destacar la producción de lesiones en la mucosa que recubre la superficie interna del tubo digestivo, en especial del estómago y duodeno, provocando la aparición de erosiones o úlceras que pueden llegar a sangrar, causando una hemorragia digestiva, o incluso a perforar la pared del órgano donde asientan. Esto se produce porque debilitan unas proteínas (enzimas) esenciales para mantener intacta la mucosa digestiva y para otras funciones del organismo como la función renal. Por ello, también pueden alterar la función de los riñones en algunos pacientes que tienen alguna enfermedad pre-existente como por ejemplo la cirrosis hepática. Cabe resaltar que la aparición de erosiones y úlceras puede producirse independientemente de cuál sea la vía de administración del fármaco (oral, inyectable o rectal) y que su toma por vía oral junto con alimentos o después de las comidas no protege de su aparición. No obstante, el riesgo no es igual para todas las personas, depende de la dosis, de la duración del tratamiento, de la utilización de otras medicaciones concomitantes, entre otros.

A demás es importante mencionar que no todos los AINES tienen el mismo riesgo de producir estos efectos secundarios y que recientemente se están incorporando nuevos fármacos diseñados para que no lesionen la mucosa digestiva, estos son los llamados "AINES selectivos" 


\section{Hemorragia digestiva alta asociada a AINES}

Vol. 3, núm. 3 Esp., (2019)

Karla Lissette Flores Flores; Luis Eduardo López Ávila; Linda Beatriz Dávila Solorzan; Stalin

Josue Alvarado Fiallo

o Coxibs. (Federacion Española del Aparato Digestivo, 2019)

Efectos secundarios de los AINES a nivel gastrointestinal

El principal mecanismo causante de los efectos secundarios del tracto GI superior asociados a los AINES es la inhibición sistémica de la síntesis de prostaglandinas, que están implicadas en la reparación y defensa de la mucosa intestinal, aunque hay otros mecanismos asociados. El efecto neto de los efectos de los AINES en la mucosa gastroduodenal es alterar la barrera mucosa protectora frente al ácido gástrico. Este ácido, junto con la acción corrosiva de la pepsina, potencia el daño mucoso gástrico iniciado por el efecto de la inhibición de prostaglandinas. El ácido gástrico desempeña así un papel fundamental en el daño gastroduodenal asociado a los AINES. La elevación del pH intragástrico por encima de 4 es, por tanto, clave en el tratamiento y prevención de los efectos secundarios del tracto GI superior vinculados al uso de AINES. Hasta un 50-60\% de los pacientes tratados con AINES pueden desarrollar algún tipo de efecto secundario a nivel GI. La toxicidad gastrointestinal de los AINES puede categorizarse en tres grupos diferenciados con repercusiones clínicas diferentes: 1). lesiones de la mucosa diagnosticadas mediante endoscopia, aun siendo asintomáticas; 2). síntomas como dispepsia, pirosis, náuseas, vómitos y dolor abdominal; y 3). complicaciones serias que precisan hospitalización, tales como hemorragia, perforación, obstrucción o el sangrado. La frecuencia de las lesiones endoscópicas es alta pero la relevancia clínica de ellas es limitada y dudosa. Se estima que entre 15 a un $40 \%$ de los pacientes que toman AINES experimentan dispepsia. Sin embargo, este frecuente efecto adverso no es un buen indicador de la posibilidad de desarrollar complicaciones gastrointestinales serias, tales como las hemorragias digestivas, De hecho, el 50-60\% de los pacientes que desarrollan una úlcera o una complicación 


\section{Hemorragia digestiva alta asociada a AINES}

Vol. 3, núm. 3 Esp., (2019)

Karla Lissette Flores Flores; Luis Eduardo López Ávila; Linda Beatriz Dávila Solorzan; Stalin Josue Alvarado Fiallo

grave y no presentan ningún síntoma ni signo previo. Finalmente, hasta un 1,5\% de los pacientes que toman AINES tradicionales desarrollarán una complicación grave y un 5\% de ellos fallecerán por esta causa. El riesgo relativo de hemorragia digestiva (HD) varía entre los diversos AINES, apreciándose que en el caso del celecoxib (selectivo) es comparable al de aquellos pacientes sin tratamiento $\mathrm{y}$, mientras productos como aceclofenaco, diclofenaco e ibuprofeno tienen un bajo riesgo relativo de $\mathrm{HD}$, otros productos como el piroxicam y el ketorolaco tienen un riesgo alto al compararlo con pacientes sin tratamiento. Muchas de estas diferencias encontradas en la práctica clínica se deben a las diferentes dosis utilizadas. Así, mientras ibuprofeno se utiliza en dosis analgésicas (menor o igual a $1200 \mathrm{mg} /$ día), piroxicam se utiliza a dosis máximas. (Lanas \& Narduli)

\section{HDA ocasionada por AINES}

La HDA puede obedecer a diversas causas y la distribución de las lesiones varía de acuerdo a la población considerada. Aproximadamente el 15\% se origina en el esófago, un $40 \%$ en el estómago y $45 \%$ en el duodeno, aunque estas cifras que pueden variar por razones geográficas y epidemiológicas. Los mecanismos involucrados en el desarrollo de HDA no están totalmente comprendidos. En el caso más común de las úlceras pépticas gastroduodenales diferentes, noxas causan daño a la mucosa y exposición de los vasos sanguíneos submucosos a la acción del ácido y la pepsina provocando la erosión y el sangrado. La alteración de la barrera mucosa suele asociase con la infección por Helicobacter pylori (HP), AINES, antiagregantes plaquetarios (AAP) e ingesta de alcohol. Uso de AINES y AAP inducen daño a la mucosa gastroduodenal por acción local disminuyendo la hidrofobicidad de la capa mucosa, aumentado la exposición al ácido y pepsina, con disfunción y daño celular y aumento de la permeabilidad 


\section{Hemorragia digestiva alta asociada a AINES}

Vol. 3, núm. 3 Esp., (2019)

Karla Lissette Flores Flores; Luis Eduardo López Ávila; Linda Beatriz Dávila Solorzan; Stalin

Josue Alvarado Fiallo

mucosa. (Liz \& Lapiedra, 2018)

Causas más comunes de HDA complicada por AINES

Las dos causas más frecuentes son la HDA por úlcera péptica y la secundaria a hipertensión portal. Al considerarse la HDA varicosa una entidad clínica diferente no será objeto de referencia en esta monografía. No obstante, a pesar de todos los procedimientos diagnósticos disponibles, hasta el 20\% de los casos de HDA quedan sin demostrar con exactitud su causa. La úlcera péptica continúa siendo la causa más frecuente de HDA, constituyendo entre el 37-50\% de los casos, siendo dos veces más frecuente el sangrado por úlcera duodenal que por úlcera gástrica. Los principales factores de riesgo para el desarrollo de úlcera péptica son la infección por HP y la administración de AINES. Ambos han demostrado ser factores de riesgo independientes para el desarrollo de la úlcera y el incremento de HDA. Sin embargo, estos factores podrían modificarse en un futuro, debido al uso creciente de la terapia erradicadora frente al HP, el desarrollo de AINES menos gastroerosivos o la administración concomitante de inhibidores de la bomba de protones (IBP), como él es omeprazol, en pacientes con ingesta crónica de AINES. Las lesiones agudas de la mucosa gástrica constituyen hasta el 20\% de las causas de HDA, aunque habitualmente presentan un sangrado leve. Los factores clínicos asociados con la existencia de estas lesiones son la toma de AINES, alcohol, o estado crítico del enfermo con ventilación mecánica, coagulopatía, lesiones neurológicas graves, politraumatizados, etc. (Martinez \& Calleja, 2005)

Frecuencia de consumo de AINES en los pacientes con hemorragia digestiva superior no variceal 


\section{Hemorragia digestiva alta asociada a AINES}

Vol. 3, núm. 3 Esp., (2019)

Karla Lissette Flores Flores; Luis Eduardo López Ávila; Linda Beatriz Dávila Solorzan; Stalin Josue Alvarado Fiallo

Los antiinflamatorios no esteroides son unos de los medicamentos más usados a nivel mundial, estimándose que más de 30 de millones de personas los consumen diariamente. Las complicaciones gastrointestinales relacionadas a su uso es la categoría más importante en cuanto a las reacciones adversas. En un estudio que realizado a 97 pacientes con hemorragia digestiva superior no variceal encontrándose que 69\% pertenecían al sexo masculino. De los 97 pacientes con hemorragia digestiva superior no variceal 57\% consumían algún tipo de AINES. Por otro lado, 64\% de los pacientes con hemorragia digestiva superior que consumían AINES tenían más de 60 años, lo cual coincide con la literatura publicada donde se ha demostrado que el riesgo de sangrado aumenta en proporción a la edad. En este estudio se evidencio que el tipo de AINE consumido con mayor frecuencia fue la aspirina (67\%), seguido del ibuprofeno en $15 \%$ datos similares a los encontrados en la literatura revisada.

El tiempo de consumo de los AINES es importante, el riesgo se incrementa sustancialmente luego de 3 meses a pesar de los cuidados necesarios. En este estudio se encontró que $33 \%$ de los pacientes habían consumido AINES durante meses. Llama la atención que el antecedente patológico más frecuente fue la hipertensión arterial en los pacientes que recibieron AINES. Es posible que en algunos de estos casos se haya indicado aspirina como prevención de complicaciones cardiovasculares. En cuanto a la importancia se ha señalado que incluso dosis bajas de aspirina, de 100 mg/día, se asocian a un número mayor de complicaciones digestivas. En cuanto a los hallazgos endoscópicos más frecuentes en los pacientes consumidores de AINES con hemorragia digestiva superior, la ulcera gástrica estuvo presente en $57 \%$ de los pacientes, seguido de gastritis erosiva en $23 \%$ y de ulcera duodenal en $16 \%$.

Finalmente se recomienda evitar el uso indiscriminado de AINES, promoviendo las 


\section{Hemorragia digestiva alta asociada a AINES}

Vol. 3, núm. 3 Esp., (2019)

Karla Lissette Flores Flores; Luis Eduardo López Ávila; Linda Beatriz Dávila Solorzan; Stalin

Josue Alvarado Fiallo

indicaciones específicas a través de guías; seleccionar a los pacientes con alto riesgo de hemorragia asociada a AINES para indicar medidas preventivas reconocidas y hacer conciencia de que aún la "profilaxis cardiaca" con aspirina en baja concentración, puede traer consecuencias no deseadas. (Salazar, et al., 2009)

Factores de riesgo para las complicaciones gastrointestinales por AINES

Factores dependientes del paciente: edad mayor de 60 años, antecedente de enfermedad ulcerosa, antecedente de complicaciones gastrointestinales (sangrado, perforación, estenosis), enfermedad concomitante grave (reumatológica, cardiovascular, insuficiencia renal o hepática, alteraciones de coagulación)

$>$ Factores dependientes de fármacos: tipo utilizado, uso prolongado o dosis altas de AINE, combinación de dos o más, incluido el ácido acetilsalicílico (AAS), consumo concomitante de esteroides, anticoagulante, antiagregante plaquetario (clopidogrel).

$>$ Factores muy poco relacionados: H. Pylori, tabaquismo, ingestión de alcohol y otras comorbilidades.

Diversos estudios muestran que el daño gastrointestinal por AINES es dependiente de la dosis, aunque una revisión sistemática muestra que aun el AAS a dosis bajas incrementa el riesgo de san-grado gastrointestinal. El uso conjunto de otros medicamentos, como anticoagulantes, antiagregantes plaquetarios (clopidogrel), AAS, esteroides e inhibidores de recaptura de serotonina, también elevan el riesgo de lesión. (Noble, 2010)

Un efecto adverso grave y frecuente 


\section{Hemorragia digestiva alta asociada a AINES}

Vol. 3, núm. 3 Esp., (2019)

Karla Lissette Flores Flores; Luis Eduardo López Ávila; Linda Beatriz Dávila Solorzan; Stalin Josue Alvarado Fiallo

HDA es, con una gran diferencia, el efecto indeseado más grave y frecuente de los AINES. Éstos causan 150 casos de HDA por millón de habitantes al año, mientras que dan lugar, por ejemplo, por millón de habitantes al año, a menos de 0,5 casos de agranulocitosis y de anemia aplásica, menos de 0,5 de síndrome de Stevens-Johnson o necrólisis epidérmica tóxica, menos de 3 de reacciones de hipersensibilidad alérgica grave, y menos de 2 de hepatitis tóxica. El número de casos de insuficiencia cardíaca producida o descompensada por AINES es más alto, pero seguramente inferior al de HDA. (Aguilera, Agustí, Bosch, Danés, \& Llop, 2004)

Los AINES suponen el 4\% de todas las prescripciones farmacéuticas. La Food and Drug Administration (FDA) (en español la Administración de Alimentos y Medicamentos) americana ha documentado que son responsables del 20 al 25\% de todas las reacciones adversas a drogas que se comunican espontáneamente. Una cuarta parte de esas reacciones adversas corresponden a efectos gastrointestinales. Como consecuencia, un $10 \%$ de los pacientes tiene que suspender el consumo de fármaco. Cada vez se están detectando más efectos adversos por estos fármacos. Algunos son tan suficientemente importantes y frecuentes que han motivado su retirada (p. ej., droxicam). Ningún AINES es completamente seguro. (Domingo, 2002)

Las lesiones agudas de la mucosa gástrica constituyen el 20\% de las causas de HDA y se asocian a la toma de AINES, alcohol, o estado crítico del enfermo con ventilación mecánica, coagulopatía, lesiones neurológicas graves, politraumatizados, etc. La úlcera péptica continúa siendo la causa más frecuente de HDA, constituyendo entre el 37-50\% de los casos, siendo dos veces más frecuente el sangrado por úlcera duodenal que por úlcera gástrica. La magnitud del sangrado se ha correlacionado con el tamaño $(>2 \mathrm{~cm})$ y la localización de la úlcera, debido a la erosión de grandes vasos, principalmente las localizadas en cara posterior del bulbo duodenal 


\section{Hemorragia digestiva alta asociada a AINES}

Vol. 3, núm. 3 Esp., (2019)

Karla Lissette Flores Flores; Luis Eduardo López Ávila; Linda Beatriz Dávila Solorzan; Stalin

Josue Alvarado Fiallo

(arteria gastroduodenal) y en la porción proximal de la curvatura menor gástrica (arteria gástrica izquierda). Los principales factores de riesgo para el desarrollo de úlcera péptica son la infección por Helicobacter pylori y la administración de antinflamatorios no esteroideos (AINE). (Arap, Mederos, Garcia, \& Gegato)

\section{Conclusiones.}

La aplicación de métodos de vigilancia intensiva por personal capacitado resulta fundamental para el desarrollo y fortalecimiento del fármaco vigilancia (FV) y la sensibilización y educación de los profesionales de la salud y los pacientes. El énfasis debe ser puesto en el desarrollo de estrategias que apunten a promover un uso racional de los medicamentos incorporando la FV como una herramienta que contribuya al monitoreo e individualización de la terapéutica. (Olmos, Daners, Olmos, \& Giachetto, 2011)

Para los usuarios de AINES, el riesgo depende sobre todo del fármaco y de su dosis, así como de la comorbilidad y los fármacos tomados de manera concomitante. Nuestro estudio ha confirmado los riesgos elevados ya conocidos asociados a ketorolaco, piroxicam, indometacina, ketoprofeno, naproxeno y ácido acetilsalicílico no cardiovascular, e indica que incluso a dosis bajas estos fármacos se asocian a riesgos relativos elevados de HDA. (Aguilera, Agustí, Bosch, Danés, \& Llop, 2004)

Como consecuencia, dada la alta frecuencia de la asociación de ingesta de AINES y HDA aguda, parece prudente que los especialistas sean más cautos en la indicación de AINES, descartar el alcoholismo y la hipertensión arterial y confirmar que no existe antecedente ulceroso. En todo caso considerar la posibilidad de adicionar medicamentos que inhiben la 


\section{Hemorragia digestiva alta asociada a AINES}

Vol. 3, núm. 3 Esp., (2019)

Karla Lissette Flores Flores; Luis Eduardo López Ávila; Linda Beatriz Dávila Solorzan; Stalin Josue Alvarado Fiallo

secreción de ácido y que son protectores de la mucosa digestiva. Como alternativa a estos efectos indeseados usar nuevas drogas anti-inflamatorias que tienen menor efecto dañino sobre la mucosa gastrointestinal. (Roblero \& Valenzuela, 2002)

\section{Bibliografía.}

Aguilera, C., Agustí, A., Bosch, M., Danés, I., \& Llop, r. (Mayo-Junio de 2004). Antiinflamatorios no esteroides y hemorragia digestiva. Butlletí groc, 17(3), 9-12.

Aranguren, I., Elizondo, G., \& Azparren, A. (2016). Consideraciones de seguridad de los aines. Boletin de informacion farmacoterapeutica de navarra, 24(2), 1-13.

Arap, J., Mederos, O., Garcia, J., \& Gegato, A. (s.f.). Sangramiento digestivo alto. La habana. $\begin{array}{lllllll}\text { Recuperado } & \text { el } & 12 & \text { de } & 10 & \text { de } & 2019,\end{array}$ http://www.sld.cu/galerias/pdf/uvs/cirured/sda_supercurso_1.pdf

Bai, J., \& Smecuol, E. (12 de Mayo de 2004). Hemorragia digestiva alta en relación con la administración crónica de AINEs. Recuperado el 12 de 10 de 2019, de IntraMed: https://www.intramed.net/contenidover.asp?contenidoid=29198

Castellanos, M., Solis, U., Faure, A., \& Villaurrutia, Y. (2014). Gastropatía por antinflamatorios no esteroideos en pacientes con enfermedades reumáticas. Revista cubana de Reumatologia, 16(3), 356-364.

Domingo, J. (Julio de 2002). Gastropatía por AINE. Efectos adversos. Farmacia Profesional, 16(7), 48-53.

Federacion Española del Aparato Digestivo. (09 de Octubre de 2019). Alteraciones digestivas por antiinflamatorios no esteroideos. Recuperado el 12 de 10 de 2019, de FEAD: https://www.saludigestivo.es/enfermedades-digestivas-y-sintomas/alteracionesdigestivas-antiinflamatorios-no-esteroideos/

Franco, M. (s.f.). Gastropatia por AINES: Lesion gastrica, insidencias y mecanismos. Sociedad española del dolor, 4, 23-27.

Galvan, F., \& Neri, E. (2006). Efectos gastrointestinales graves por antiinflamatorios no esteroideos. Un problema no resuelto. Reumatologia clinica, 2(5), 261-271.

Lanas, A., \& Narduli, G. (s.f.). Lesiones gastrointestinales por AINE: lesiones y medidas de prevencion. El medico, 1-39. 


\section{Hemorragia digestiva alta asociada a AINES}

Vol. 3, núm. 3 Esp., (2019)

Karla Lissette Flores Flores; Luis Eduardo López Ávila; Linda Beatriz Dávila Solorzan; Stalin

Josue Alvarado Fiallo

Liz, A., \& Lapiedra, D. (2018). Hemorragia digestiva alta. Clínicas Quirúrgicas Facultad de Medicina Universidad de la República. Uruguay, 1-7.

Martinez, J., \& Calleja, J. (2005). Hemorragia digestiva alta: etiología y procedimientos y diagnostico. Emergencias, 17, 50-57.

Noble, A. (2010). Lesión gastrointestinal por AINE/COXIB y su tratamiento. Revista de Gastroenterología de México, 1(75), 22-25.

Olmos, I., Daners, M., Olmos, V., \& Giachetto, G. (2011). Consultas por sangrados relacionados con medicamentos en el Servicio de Emergencia de la Asociación Española Primera de Socorros Mutuos. Revista medica de Uruguay, 27(1), 5-11.

Roblero, J., \& Valenzuela, J. (Enero de 2002). Hemorragia Digestiva Alta y Anti-Inflamatorios no Esteroidales: Una Experiencia Local. Revista Médica Clínica Las Condes, 13(1), 1-7.

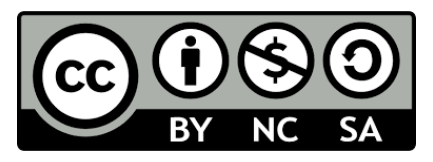

RECONOCIMIENTO-NOCOMERCIAL-COMPARTIRIGUAL

CC BY-NC-SA

ESTA LICENCIA PERMITE A OTROS ENTREMEZCLAR, AJUSTAR Y CONSTRUIR A PARTIR DE SU OBRA CON FINES NO

COMERCIALES, SIEMPRE Y CUANDO LE RECONOZCAN LA AUTORÍA Y SUS NUEVAS CREACIONES ESTÉN BAJO UNA LICENCIA CON LOS MISMOS TÉRMINOS. 